\title{
Erratum to: Dosimetric comparison of different treatment modalities for stereotactic radiosurgery of meningioma
}

\author{
David Kaul • Harun Badakhshi • Thierry Gevaert • Diana Pasemann • \\ Volker Budach • Constantin Tuleasca • Arne Gruen • Vikas Prasad • \\ Marc Levivier • Markus Kufeld
}

Published online: 23 December 2014

(C) Springer-Verlag Wien 2014

\section{Erratum to: Acta Neurochir}

DOI 10.1007/s00701-014-2272-9

The original version of this article unfortunately contained mistakes.

The name of Constantin Tulaesca is misspelled, it should be Constantin Tuleasca.

The correct affiliation of C. Tuleasca and M. Levivier should be:
Lausanne University Hospital, Neurosurgery Service and Gamma Knife Center; University of Lausanne, Faculty of Biology and Medicine

On the third page, Subtitle "Patient population", lines 18 and 19:

"Gamma Knife planning and optimalisation was performed by Thierry Gevaert"

should be: "Gamma Knife planning and optimalisation was performed by Marc Levivier".

The online version of the original article can be found at http://dx.doi.org/ 10.1007/s00701-014-2272-9.

D. Kaul $(\bowtie) \cdot$ H. Badakhshi $\cdot$ D. Pasemann $\cdot$ V. Budach · A. Gruen Department of Radiation Oncology, Charité School of Medicine and University Hospital, Campus Virchow-Klinikum, Augustenburger Platz 1, 13353 Berlin, Germany

e-mail: david.kaul@charite.de

T. Gevaert

Department of Radiotherapy, Universitair Ziekenhuis Brussel,

Brussels, Belgium

C. Tuleasca $\cdot$ M. Levivier

Lausanne University Hospital, Neurosurgery Service and Gamma

Knife Center, University of Lausanne, Faculty of Biology and

Medicine, Lausanne, Switzerland

M. Kufeld

Charité CyberKnife Center, Charité School of Medicine and

University Hospital, Campus Virchow-Klinikum, Augustenburger

Platz 1, 13353 Berlin, Germany

\section{Prasad}

Department of Nuclear Medicine, Charité School of Medicine and University Hospital, Campus Virchow-Klinikum, Augustenburger

Platz 1, 13353 Berlin, Germany 